\title{
Enhanced B7 costimulatory molecule expression in inflammatory human sural nerve biopsies
}

\author{
R Kiefer, F Dangond, M Mueller, K V Toyka, D A Hafler, H-P Hartung
}

Klinik und Poliklinik für Neurologie, Westfälische

Wilhelms-Universität, Albert-SchweitzerStrasse 33, D-48129

Münster, Germany

R Kiefer

M Mueller

Center for Neurologic Diseases and

Department of

Neurology, Brigham

and Women's Hospital,

Harvard Medical

School, Boston, USA

F Dangond

D A Hafler

Department of

Neurology, Bayerische

Julius-Maximilians-

Universität,

Würzburg, Germany

K V Toyka

Department of

Neurology,

Karl-Franzens-

Universität, Graz,

Austria

H-P Hartung

Correspondence to:

Dr Reinhard Kiefer

kieferr@uni-muenster.de

Received 19 November 1999 and in revised form

24 February 2000

Accepted 6 March 2000

\begin{abstract}
Objectives-To define the role of the costimulatory molecules $B 7-1$ and $B 7-2$ in inflammatory disorders of the peripheral nervous system. B7 molecules are essential for effective antigen presentation and may determine the differentiation of $T$ cells into a Th-1 or Th-2 phenotype, thus modulating immune response and disease course.

Methods-Forty nine sural nerve biopsies from patients with neuroborreliosis, Guillain-Barré syndrome (GBS), chronic inflammatory demyelinating polyneuropathy (CIDP), CIDP variants and hereditary neuropathies, and those with no detectable abnormality were investigated. The expression of B7-1 and B7-2 mRNA and protein was investigated by polymerase chain reaction (PCR) and immunocytochemistry.

Results-B7-1 mRNA was strongly upregulated in both cases of neuroborreliosis, in two cases of GBS and one case of variant CIDP. Moderate to low levels were detected in the remaining GBS and CIDP biopsies and were rarely found in a noninflammatory control group consisting of hereditary neuropathy and normal nerves. At the immunocytochemical level, strong expression of $\mathrm{B} 7-1$ protein was found in both neuroborreliosis cases, and moderate or low expression in six of eight GBS cases and seven of 17 CIDP cases investigated, whereas only one of five non-inflammatory control nerves showed staining, which was very weak. In neuroborreliosis, B7-1 protein was found very pronounced in epineurial infiltrates, whereas in GBS and CIDP, labelling was predominantly endoneurial and localised to putative macrophages. B7-2 mRNA and protein were expressed only at low levels in neuroborreliosis and selected autoimmune neuropathy cases, and were essentially absent from noninflammatory controls.

Conclusions-B7 molecules are expressed in the peripheral nervous system and regulated during disease, and their presence in macrophages underlines the putative function of endoneurial macrophages as local antigen presenting cells in the immunopathology of peripheral nerve. B7-1 rather than $B 7-2$ is preferentially upregulated, possibly promoting the induction of a Th-1-type $T$ cell response within the nerve.

(F Neurol Neurosurg Psychiatry 2000;69:362-368)
\end{abstract}

Keywords: B7; antigen presentation; peripheral neuropathy; macrophage
Guillain-Barré syndrome (GBS) and chronic inflammatory demyelinating polyneuropathy (CIDP) are inflammatory disorders of presumed autoimmune aetiology of the peripheral nervous system (PNS). Activated autoreactive $\mathrm{T}$ cells are probably of key importance in the initiation of disease ${ }^{1}$ although humoral immune responses play a part in pathogenesis. ${ }^{23}$ Activation of $\mathrm{T}$ cells not only requires presentation of processed immunogenic peptide fragments on the surface of local antigen presenting cells in the context of major histocompatibility complex (MHC) antigens to interact with the $\mathrm{T}$ cell receptor, but also additional antigen-non-specific costimulatory signals. One of these signals is provided through the interaction of the costimulatory molecules B7-1 and B7-2 on antigen presenting cells with their ligands CD28 and CTLA-4. ${ }^{45}$ Antigen presentation in the presence of $\mathrm{B} 7$ results in $\mathrm{T}$ cell activation whereas absence of signals from B7 molecules may induce anergy or immunosuppression. Furthermore, antigen presentation in the presence of B7-1 may drive $\mathrm{T}$ cells towards the Th-1 phenotype with expression of interleukin (IL)-2, interferon (IFN) $-\gamma$, and tumour necrosis factor (TNF) $-\alpha$ whereas presentation in association with B7-2 may induce the Th-2 phenotype with predominant expression of IL-4, ${ }^{67}$ although recent in vitro experiments using antigen presenting cells deficient in B7-1 or B7-2 give less clear results. $^{8}$ Finally, whereas interaction with CD28 on T cells results in activation, engagement of CTLA-4 may deliver an inhibitory signal to $\mathrm{T}$ cells and induce peripheral $\mathrm{T}$ cell tolerance. ${ }^{9}$

The expression of B7 molecules is not restricted to monocytes and macrophages but occurs on other immune cells including dendritic cells, $\mathrm{T}$ cells, and $\mathrm{B}$ cells. ${ }^{4}$ In the CNS, microglial cells are the predominant cell type expressing B7 molecules. ${ }^{10}{ }^{11}$ In multiple sclerosis lesions, immune cells including putative microglial cells express B7-1 and B7-2, ${ }^{10-12}$ whereas in stroke B7-2 is exclusively expressed. In vitro, human microglia constitutively express B7-2 and display B7-1 with prolonged culture in a time dependent manner. ${ }^{13-15}$ Both molecules can also be upregulated on cytokine stimulation in vitro. By virtue of their ability to express B7, microglia may act as local antigen presenting cells in the brain and may play a critical part in lesion formation in multiple sclerosis. In the PNS however, the function of B7 molecules has not been defined. In sural nerve biopsies from patients with inflammatory and non-inflammatory neuropathies, MHC class II antigens have been found both on 
Summary of patient characteristics and availability of material for polymerase chain reaction (PCR) experiments and immunohistochemistry (IHC)

\begin{tabular}{llllll}
\hline Diagnosis & No of cases & Age $(y)$ & Duration of disease & $\begin{array}{l}\text { No of biopsies } \\
\text { available for PCR }\end{array}$ & $\begin{array}{l}\text { No of biopsies } \\
\text { available for IHC }\end{array}$ \\
\hline Neuroborreliosis & 2 & 39 and 52 & 1 and 2 months & 2 & 2 \\
GBS & 12 & $21-73$ & $3-18$ days & 11 & 8 \\
CIDP & 20 & $22-74$ & 3 months-22 y & 18 & 17 \\
CIDP variants & 8 & $32-78$ & 2 months-5 y & 8 & 4 \\
Hereditary neuropathies & 5 & $24-80$ & Not applicable & 5 & 1 \\
Normal nerves & 2 & 33 and 45 & Not applicable & 1 & \\
\hline
\end{tabular}

GBS=Guillain-Barré syndrome; CIDP=chronic inflammatory demyelinating polyneuropathy.

macrophages and Schwann cells, ${ }^{16}$ whereas in experimental animals, macrophages seem to be the major site of MHC II expression. ${ }^{17}{ }^{18}$ Thus macrophages rather than Schwann cells are the likely antigen presenting cell in the PNS and, in addition to executing cytotoxicity towards myelin sheaths, they may be critical components in initiating an autoimmmune response such as in GBS and CIDP. ${ }^{1}$ To date it is unknown whether and where B7 molecules are expressed in peripheral nerve and how the local microenvironment of axons and Schwann cells may influence B7-1 and B7-2 preponderance. We have therefore investigated the presence of B7-1 and B7-2 mRNA and protein in sural nerve biopsies from patients with inflammatory and non-inflammatory neuropathies.

\section{Methods}

PATIENTS

Sural nerve biopsies from 49 patients were examined. They were performed for diagnostic purposes with informed consent, and scientific investigations on these biopsies were performed under a research protocol approved by the University of Würzburg ethics committee. Most of these nerves were investigated in previous studies, and details of the patients were described elsewhere. ${ }^{19}{ }^{20}$ The clinical data and the availability of biopsy material for polymerase chain reaction (PCR) experiments and immunohistochemistry are given in the table. In brief, two patients had definite acute neuroborreliosis and harboured borrelia burgdorferi IgM antibodies in their serum and CSF. Twelve patients had the demyelinating form of acute GBS. ${ }^{21}$ These biopsies were taken in the context of a study protocol. Twenty patients had CIDP as diagnosed by accepted research criteria $^{22}$ with minor modifications as described. ${ }^{19}$ Four of these patients had additional diagnoses that might possibly relate to peripheral nerve damage, including stenosis of the lumbar spinal canal, lymphoma, diabetes mellitus, and chronic infection, and one patient was biopsied twice during the course of his disease. Eight patients had variants of CIDP that either formally differed from classic CIDP by the absence of motor symptoms and signs or fulfilled only part of the diagnostic criteria of CIDP but featured marked inflammatory infiltrates in sural nerve biopsies. ${ }^{19}$ Five biopsies were available from patients with hereditary neuropathies including hereditary sensory neuropathy, hereditary motor and sensory neuropathy type II, hereditary neuropathy with liability to pressure palsies (two cases), and mitochondrial neuropathy. Finally, two biop- sies seemed normal and were taken from clinically and electrophysiologically normal nerves for suspected cerebral vasculitis and metabolic encephalopathy. The normal biopsies and the biopsies from patients with hereditary neuropathies were grouped together to serve as non-inflammatory controls.

RNA EXTRACTION AND PCR ANALYSIS

Biopsy specimens of $2-3 \mathrm{~mm}$ in length were homogenised and total RNA was extracted according to standard procedures as described previously. Three tenths of the resulting total RNA was reversely transcribed using SuperscriptII reverse transcriptase (Promega, Madison, WI, USA) and $25 \mathrm{ng} / \mu \mathrm{l}$ oligo(dT) 12-18 and random hexamers as primers (both from Pharmacia, Uppsala, Sweden). One twentieth ( $\beta$-actin) or one eighth (B7-1 and B7-2) of the resulting first strand cDNA was amplified by PCR using $0.5 \mu \mathrm{g}$ each of forward and reverse primer, $80 \mathrm{nM}$ dNTP, 1,2 U Taq polymerase (Perkin Elmer, Foster City, CA, USA) and 0.08 $\mu \mathrm{l}\left({ }^{32} \mathrm{P}\right) \mathrm{dCTP}(3000 \mathrm{Ci} / \mathrm{mmol}$; Amersham, Little Chalfont, England) in a total volume of 30 $\mu 1$. Amplification was conducted for 33 cycles (B7-1 and B7-2) or 30 cycles ( $\beta$-actin), each of 60 seconds at $94^{\circ} \mathrm{C}$ for denaturation, 60 seconds at $60^{\circ} \mathrm{C}$ for annealing, and 90 seconds at $72^{\circ} \mathrm{C}$ for extension. For B7 amplification, the following primers were used: ${ }^{12}{ }^{15} 5$-AAC TCG CAT CTA CTG GCA AAA GGA GAA-3' and 5'-GGG AAA CTG TTG TGT TGA TGG CAT TTA-3' for B7-1; 5'-TGA TTC GGA CAG TTG GAC CCT GAG AC-3' and 5'-CTT GTC AGT TTC CAG AAT ACA GAA GAT GGT C-3' for B7-2. After amplification, the samples were subjected to $5 \%$ polyacrylamide gel electrophoresis, the gels were dried, and PCR products were visualised by autoradiography on Fuji RX $x$ ray film for 2-24 hours. Quantification of amplification products was achieved using a phosphorimager Betascope 603 Blot Analyzer (Betagen, Waltham, MA, USA). B7 mRNA content was expressed in arbitrary units as the mean of two independent experiments and semiquantitatively assessed after normalisation for $\beta$-actin mRNA content. In some experiments, specificity of non-radioactive PCR products was confirmed by Southern blotting and hybridisation with radioactively labelled internal oligonucleotides (for B7-1: 5'-AGA AAA TGG TGC TGA CTA TGA TGT CTG-3'; for B7-2: 5'-GTA TCA ATG TAT CAT CCA TCA CAA AAA GCC C-3'). 


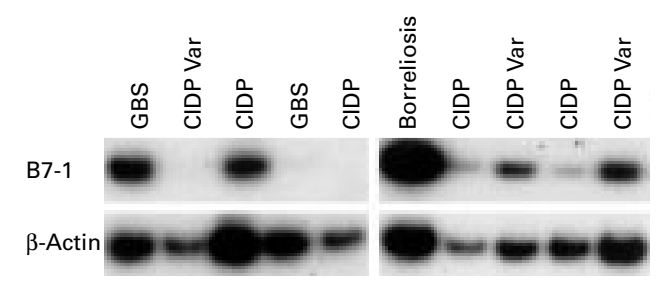

Figure 1 Representative PCR experiment to detect B7-1 $m R N A$ in nerve biopsies showing upregulation of B7-1 $m R N A$ in selected cases with inflammatory neuropathies. Variability of $\beta$-actin signal intensity is due to different amounts of tissue in the biopsy samples.

IMMUNOCYTOCHEMISTRY

Ten micron thick cryostat sections of sural nerve specimens and human tonsil as positive control tissues were air dried and fixed for 1 minute each in $3.7 \%$ phosphate buffered formalin and 50\%, $100 \%$, and $50 \%$ acetone at room temperature. After blockade of non-specific binding with 10\% bovine serum albumin, primary antibodies were applied overnight at $4^{\circ} \mathrm{C}$ in a humid chamber. Primary mouse anti human B7-1 and B7-2 antibodies, a gift from Dr Vijay Kuchroo, Harvard Medical School, were used at $5 \mu \mathrm{g} / \mathrm{ml}$ for purified antibody and 1:25 for hybridoma supernatant. Bound antibody was detected using secondary biotinylated antimouse antibody and avidin biotinylated peroxidase complex, both at 1:100 for 1 hour at room temperature (Dako, Hamburg, Germany), and 3,3'-diaminobenzidine as chromogen. Sections treated without primary antibody or equivalent concentrations of an irrelevant antibody served as negative controls. For semiquantitative assessment, the number and intensity of the immunocytochemical signals in the biopsy specimens was graded as strong (grade 3), moderate (grade 2 ), weak (grade 1), or absent (grade 0) without knowledge of the PCR results.

\section{Results}

DETECTION OF B7-1 AND B7-2 MRNA BY PCR

Polymerase chain reaction amplification of cDNA derived from sural nerve biopies demonstrated expression of B7-1 mRNA in a subgroup of biopsy specimens (fig 1). However, the degree of B7-1 mRNA expression showed marked variation between individual biopies. A semiquantitative estimate, relating the amount of B7 PCR product to that of actin, disclosed strong B7-1 amplification signals in both cases of established neuroborreliosis, two of the 11 GBS cases, and in one of the eight cases with CIDP variant. Moderate to low expression was found in the remaining cases with GBS, CIDP (20 cases), and CIDP variants, and the non-inflammatory control group of normal and hereditary neuropathies (seven cases), and was virtually absent in some (fig $2 \mathrm{~A}$ ).

By contrast, B7-2 mRNA was only rarely expressed. Low levels of B7-2 amplification products were found in both cases with neuroborreliosis, two of 11 cases with GBS, three of 20 cases with CIDP and four of the eight CIDP variant cases. No expression was found in the remaining cases including all seven patients with hereditary neuropathies or no abnormality (fig $2 \mathrm{~A}$ ).

\section{IMMUNOCYTOCHEMICAL DETECTION OF B7-1 AND} B7-2

Twenty three monoclonal antibodies against human B7-1 and B7-2 were tested for their usefulness in immunocytochemistry. Seven out of 14 antibodies directed against B7-1 and two out of nine antibodies directed against B7-2 consistently labelled round cells in human tonsils serving as positive control tissue, ${ }^{23}$ with comparable staining patterns
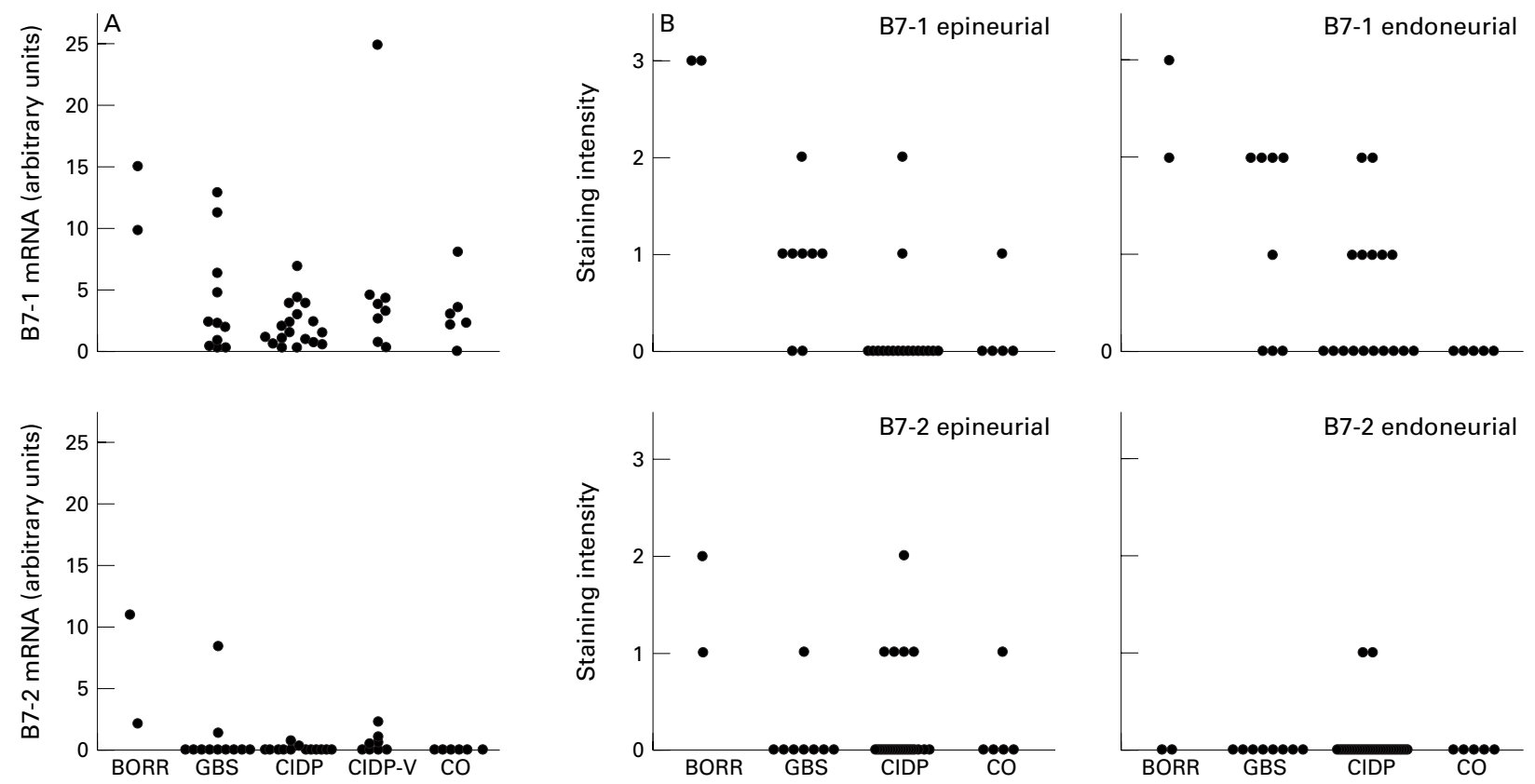

Figure 2 (A) Semiquantitative estimation of B7-1 and B7-2 $m R N A$ content in sural nerve biopsies of two cases with neuroborreliosis (BORR), 11 cases of Guillain-Barré syndrome (GBS), 18 cases of chronic inflammatory demyelinating polyneuropathy (CIDP), eight CIDP variants (CIDP-V), and six non-inflammatory controls (CO). Results are mean values of two independent experiments normalised for $\beta$-actin mRNA content. (B) Semiquantitative estimation of B7-1 and B7-2 immunocytochemical staining intensities in sural nerve biopsies of two cases with neuroborreliosis, eight cases of GBS, 17 cases of CIDP, and six non-inflammatory controls. Grading was grade $3=$ strong, grade $2=$ moderate, grade $1=$ weak, and grade $0=a b s e n t$. 

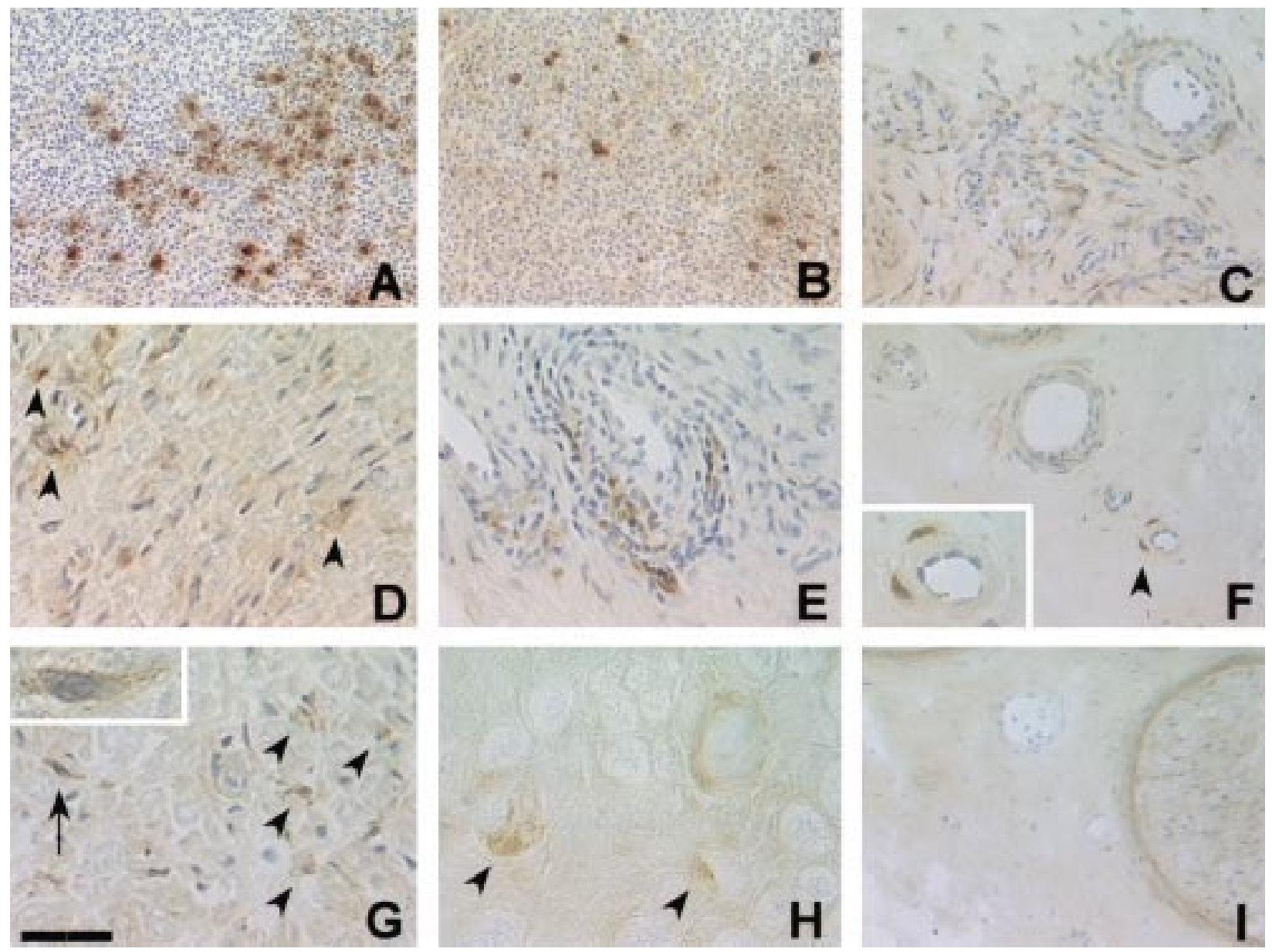

Figure 3 Immunocytochemistry for $B 7$ in $(A, B)$ human tonsil and $(C-I)$ various neuropathies. Human tonsil served as positive control tissue, disclosing numerous (A) B7-1 and (B) B7-2 positive mononuclear cells. (C-E) In neuroborreliosis, B7-1 expression was found in (C) numerous epineurial cells and to a lesser extent in (D, arrowheads) the endoneurium. Many of the epineurial B7-1 positive cells were ramified and resembled macrophages by morphology and distribution, whereas endoneurial labelled cells were rounded or elongated and again were distributed similar to macrophages. (E) B7-2 expression was restricted to epineurial perivascular round cells in fewer areas. $(F, G)$ In Guillain-Barré syndrome, there was only weak expression in rare epineurial perivascular round cells (F, arrowhead and inset), whereas numerous endoneurial labelled cells were found (G, arrowheads). These cells were distributed like macrophages, and carried small ramifications or were elongated with the typical morphology of endoneurial macrophages ( $G$, arrow and inset) and distinct from Schwann cells. ( $H$, arrows) A similar, but often very weak labelling pattern was found in isolated cases of chronic inflammatory demyelinating

polyneuropathy whereas no unequivocal staining was found in non-inflammatory control nerves (I, hereditary neuropathy with liability to pressure palsies). In such cases, only background staining was found. Technical controls were negative throughout (not shown). All sections were counterstained with haematoxylin except H. Bar $=100 \mu \mathrm{m}$ in A-C and I, $50 \mu \mathrm{m}$ in D-F, $35 \mu \mathrm{m}$ in $\mathrm{G}$, and $20 \mu \mathrm{m}$ in $\mathrm{H}$.

(fig 3 A, B). Control sections after omission of primary antibody or use of an irrelevant antibody were always negative both on tonsil and nerve.

B7-1 was strongly expressed in both cases with neuroborreliosis. Intense immunocytochemical reaction product was found in ramified cells in the epineurium often located within perivascular round cell infiltrates (fig 2 $\mathrm{B}$ and fig $3 \mathrm{C}$ ). Endoneurial expression was also marked but somewhat weaker and present in scattered cells throughout the endoneurium (fig $3 \mathrm{D}$ ). By morphology and distribution, these cells strongly resembled endoneurial and epineurial macrophages rather than $\mathrm{T}$ cells or other cellular components of the peripheral nerve. Immunocytochemical stains on neighbouring sections for $\mathrm{T}$ cells and macrophages confirmed similar staining patterns betweeen B7-1 and macrophages, although fewer cells were stained with B7-1 antibody than with macrophage markers (not shown).

In six of eight GBS cases and seven of 17 CIDP cases investigated, B7-1 signals were detected but were weaker than in neuroborreliosis (fig 2 B). Expression in GBS was generally stronger than in CIDP. There was limited expression on scattered epineurial perivascular cells (fig $3 \mathrm{~F}$ ), with occasional perivascular clusters of B7-1 positive cells. In the endoneurium, there consistently were numerous scattered cells with small ramifications or an elongated shape resembling endoneurial macrophages that were weakly or moderately labelled with B7-1 antibody (fig 3 $\mathrm{G}, \mathrm{H})$. Occasionally, rounded cell profiles in the vicinity of myelin sheaths were noted that might represent Schwann cells. Comparing the staining pattern with the neuroborreliosis cases, endoneurial staining was relatively more pronounced than epineurial staining in GBS and CIDP, whereas the opposite was true for the neuroborreliosis cases.

In the hereditary neuropathy cases studied, either no specific staining or only very occasional epineurial cells staining for B7-1 were found, with minimal or absent endoneurial staining (fig $2 \mathrm{~B}$, fig $3 \mathrm{I}$ ). 
B7-2 immunoreactivity was only very rarely found. In the neuroborreliosis cases, B7-2 was identified in round cell infiltrates (fig $3 \mathrm{E}$ ) and occasional epineurial ramified cells, with no definite staining within the endoneurium. In the other inflammatory neuropathies, no consistent endoneurial staining was demonstrable but unequivocal albeit weak staining for B7-2 was noticeable in two CIDP cases. Some labelled epineurial cells were also occasionally found which could also be seen in one of the hereditary neuropathy cases.

COMPARISON OF PCR AND

IMMUNOHISTOCHEMICAL RESULTS

Twenty seven biopsies were available both for PCR and immunohistochemistry. In 21 of these biopsies PCR and immunohistochemical data were supportive of each other. In three samples, there were B7 PCR amplificates above background but no immunohistochemical signals, and in another three biopsies we found immunohistochemical signals with low or absent B7 mRNA.

\section{Discussion}

The initiation of a local immune response in the peripheral nerve as a prerequisite for autoimmune inflammation requires effective presentation of antigen to surveying $\mathrm{T}$ cells. Local resident macrophages are main candidates for antigen presentation as they readily express MHC class II molecules both in a resting state and after activation. ${ }^{16}{ }^{18}$ We now report that the costimulatory molecules B7-1 and to a lesser extent B7-2 may also be found in peripheral nerve and are regulated during autoimmune disease. These data demonstrate that local cells within the PNS may effectively present antigen to $\mathrm{T}$ cells enabling them to actively participate in the initiation and maintenance of an autoimmune response. They also underline the apparent immunocompetence of the PNS.

B7-1 mRNA and protein were not present in all biopsies but were found at high levels mainly in an infectious neuropathy caused by Borrelia burgdorferi and in a subset of cases with GBS, CIDP, and CIDP variants, with only low levels in others and complete absence in some. In interpreting these data, it should be noted that due to the low amounts of RNA available no proper quantitative PCR with internal competitors could be performed, ${ }^{24}$ and that immunocytochemical quantification reflects no more than estimates as the stains were generally too weak to allow for reliable numerical quantification of labelled cells. However, immunocytochemical and PCR data are largely consistent and supportive of each other, although $\mathrm{mRNA}$ was occasionally found in biopsies that were negative at the immunocytochemical level, and vice versa. This might be due to differences in sensitivity or sampling errors, or due to B7 mRNA present in contaminating blood cells within the biopsy samples. It should further be noted that immunocytochemistry, although weak, was well reproduced within individual nerves providing reliable results, whereas PCR quantification was less controlled due to lack of an internal competitor. Despite these technical considerations it seems clear that B7-1 mRNA and particularly B7-1 protein are upregulated in several inflammatory neuropathies compared with subjects with normal nerves and patients with hereditary neuropathy. B7-1 expression, particularly in the epineurium, seemed to be more pronounced in inflammatory infiltrates from patients with more acute neuropathies of short duration such as neuroborreliosis and GBS. This is consistent with our findings in multiple sclerosis where B7-1 was upregulated particularly in acute plaques from early disease cases. ${ }^{12}$ However, it should be noted that the absence of B7 in other inflammatory biopsy specimens does not necessarily reflect true absence because of the well known sampling issues involved in obtaining peripheral nerve biopsy specimens. Thus B7 expression may go undetected even if present elsewhere or at another time in the same patient. In non-inflammatory controls, only minimal immunocytochemical signal for B7-1 protein was present in one single biopsy whereas it was absent in the others. Low levels of B7-1 PCR amplification product in these biopsies may again either reflect the higher sensitivity of the method or the amplification of mRNA derived from contaminating blood cells inevitably present in the specimens. In addition, inflammatory infiltrates may occasionally be present in hereditary neuropathies. Similarly, B7-2 was noted in biopsies from patients with inflammatory neuropathies but was virtually absent from non-inflammatory controls. Unfortunately, only two normal nerves were available for comparison as such nerves usually are not being biopsied.

The preferential overexpression of B7-1 in GBS and CIDP, in addition to the neuroborreliosis cases, emphasises the immunoinflammatory aetiology of these conditions. Interestingly, expression was particularly strong epineurially in neuroborreliosis and more conspicuous endoneurially in GBS and CIDP. These differences may reflect the actual site of the inflammatory response, which is an epineurial perivasculitis in neuroborreliosis ${ }^{25}$ and an immune response against myelin and other endoneurial components in the case of GBS and CIDP. ${ }^{1}$ Although published data on MHC II expression were not reproduced in the present study, it seems that B7 expression does not correlate closely with MHC II expression as MHC II positive macrophages are also abundantly present in degenerative neuropathies. MHC II expression may thus be part of a more general activation programme of macrophages, much as it is used by activated microglial cells of the CNS which readily express MHC II molecules once activated. ${ }^{26}$ By contrast, B7 expression may be more restricted and specific for inflammatory conditions where it may be required as a costimulatory signal in antigen presentation. Thus as a preliminary speculation, B7 expression might even be useful as a diagnostic marker to differentiate inflammatory neuropathies from degenerative conditions, as has been described for other inflammatory macrophage markers. ${ }^{19}$ 
Macrophages seemed to be the main source of B7 molecules in the present study. This assumption is based on the typical morphology and distribution of B7 positive cells both epineurially and endoneurially. It is additionally supported by colocalisation of B7-1 and the macrophage antigen CD 68 in some double immunofluorescence experiments not shown in the present paper as B7-1 immunofluorescence was too weak to be unequivocally reproduced and photodocumented. In addition, some occasional B7-1 positive putative Schwann cells were seen, and B7-2 positive round cells might either represent macrophages, $\mathrm{T}$ cells, or B cells. Thus, B7 expression was found on those cells already known to express B7 molecules in vitro and present antigen, ${ }^{4}$ and those expressing MHC II in peripheral nerve-namely, macrophages. ${ }^{16}{ }^{18}$ Our data thus further support the accepted notion that macrophages rather than Schwann cells are the main immunocompetent cell of the PNS.

In most biopsies, B7-1 mRNA and protein were much stronger and more widely expressed than B7-2. These findings differ somewhat from in vivo studies in multiple sclerosis where B7-2 staining was also widely found. ${ }^{12}$ In an experimental model of multiple sclerosis, experimental autoimmune encephalomyelitis, B7-2 also seemed to be more readily expressed than B7-1. ${ }^{27}$ Such differences may be important as presentation of antigen in the context of B7-1 and B7-2 may determine the type of $\mathrm{T}$ cell response to antigen-namely, Th-1 and Th-2. ${ }^{6728}$ The preferential expression of B7-1 over B7-2 particularly in neuroborreliosis and GBS may therefore indicate the evolution of a proinflammatory, primarily $\mathrm{Th}-1$ type $\mathrm{T}$ cell response, possibly consistent with the more acute course of these diseases. Indeed, a Th-1type $\mathrm{T}$ cell response has been demonstrated in Lyme arthritis, ${ }^{29}$ and Th-1-type cytokine responses are thought to be associated with disease susceptibility, ${ }^{30}$ whereas $\mathrm{T}$ cells generating both interferon (IFN) $-\gamma$ and IL-10 were found in chronic infection. ${ }^{31}$ Cytokine studies in GBS nerves are sparse, but experiments in experimental autoimmune neuritis, an animal model of GBS, have shown early expression of IFN- $\gamma$, IL-2, IL12p40, and TNF- $\alpha$, with later expression of the immunosuppressive cytokines transforming growth factor (TGF)- $\beta 1$ and IL-10. ${ }^{32-36}$ Whether B7-1 and B7-2 expression relate to specific cytokine profiles of $\mathrm{T}$ cells present in these biopsies remains to be determined. Furthermore, it is unknown whether B7 molecules in peripheral nerve would interact with CTLA-4 or CD28 in vivo. Studies in experimental autoimmune encephalomyelitis have shown a contribution of CTLA-4 to disease down regulation whereas CD28 is required for disease induction and progression. ${ }^{37}$

In summary, our data document for the first time the expression of B7 costimulatory molecules during autoimmune or infectious inflammation in human peripheral nerves and thus underline the immunocompetence of the PNS microenvironment and of endoneurial macrophages in particular. Future studies will have to further delineate the role of deviations of the B7 system in the pathogenesis of inflammatory neuropathies and to clarify whether B7 molecules may serve as therapeutic targets in the treatment of inflammatory PNS disorders.

This work was supported by grants from NIH (NS24247 to DAH), Deutsche Forschungsgemeinschaft (Ki 532/3-1), WWU Münster Medical Faculty (IMF program), Gemeinnützige Hertie-Stiftung, and University Research Funds of the University of Würzburg. We thank Dr Vijay Kuchroo, Harvard Medical School, Boston, for kindly supplying us with anti-B7-1 and anti-B7-2 monoclonal antibodies, and Heidrun Pischel and Antje Stöber for skillful technical assistance. Early parts of this study were performed while RK and HPH were at the Department of Neurology, University of Würzburg, Germany.

1 Hartung H-P, Kiefer R, Gold R, et al. Autoimmunity in the peripheral nervous system. Ballieres Clin Neurol 1996;5:1perip.

2 Enders U, Karch H, Toyka KV, et al. The spectrum of immune responses to Campylobacter jejuni and glycoconjugates in Guillain-Barre syndrome and in other neuroimmunological disorders. Ann Neurol 1993;34:13644

3 Buchwald B, Toyka KV, Zielasek J, et al. Neuromuscular blockade of IgG antibodies from patients with GuillainBarré syndrome: a macro-patch-clamp study. Ann Neurol 1998;44:913-22

4 Lenschow DJ, Walunas TL, Bluestone JA. CD28/B7 system of T cell costimulation. Annu Rev Immunol 1996;14:23358

5 Greenfield EA, Nguyen KA, Kuchroo VK. CD28/B7 costimulation: a review. Crit Rev Immunol 1998;18:389418.

6 Kuchroo VK, Das MP, Brown JA, et al. B7-1 and B7-2 costimulatory molecules activate differentially the Th1/Th2 developmental pathways: application to autoimmune disdevelopmental pathways: application
ease therapy. Cell 1995;80:707-18.

7 Racke MK, Scott DE, Quigley L, et al. Distinct roles for Racke MK, Scott DE, Quigley L, et al. Distinct roles for
B7-1 (CD-80) and B7-2 (CD-86) in the initiation of B7-1 (CD-80) and B7-2 (CD-86) in the initiation of 96:2195-203.

8 Schweitzer AN, Borriello F, Wong RC, et al. Role of costimulators in $\mathrm{T}$ cell differentiation: studies using antigen-presenting cells lacking expression of CD80 or CD86. F Immunol 1997;158:2713-22.

9 Chambers CA, Allison JP. Costimulatory regulation of T cell function. Curr Opin Cell Biol 1999;11:203-10.

10 Williams K, Ulvestad E, Antel JP. B7/BB-1 antigen expression on adult human microglia studied in vitro and in situ. Eur F Immunol 1994;24:3031-7.

11 De Simone R, Giampaolo A, Giometto B, et al. The costimulatory molecule B7 is expressed on human microglia in culture and in multiple sclerosis acute lesions. J Neuropathol Exp Neurol 1995;54:175-87.

12 Windhagen A, Newcombe J, Dangond F, et al. Expression of costimulatory molecules B7-1 (CD80), B7-2 (CD86), and interleukin 12 cytokine in multiple sclerosis lesions. $\mathcal{f}$ Exp Med 1995;182:1985-96.

13 Satoh J, Lee YB, Kim SU. T-cell costimulatory molecules B7-1 (CD80) and B7-2 (CD86) are expressed in human microglia but not in astrocytes in culture. Brain Res 1995;704:92-6.

4 Becher B, Antel JP. Comparison of phenotypic and unctional properties of immediately ex vivo and cultured human adult microglia. Glia 1996;18:1-10.

15 Dangond F, Windhagen A, Groves CJ, et al. Constitutive expression of costimulatory molecules by human microglia and its relevance to CNS autoimmunity. F Neuroimmunol 1997;76:132-8.

16 Pollard JD, McCombe PA, Baverstock J, et al. Class II antigen expression and $\mathrm{T}$ lymphocyte subsets in chronic inflammatory demyelinating polyneuropathy. $f$ Neuroimmunol 1986;13:123-34.

17 Schmidt B, Stoll G, Hartung H-P, et al. Macrophages but Schmidt B, Stoll G, Hartung H-P, et al. Macrophages but
not Schwann cells express Ia antigen in experimental not Schwann cells express Ia antigen in exp

18 Monaco S, Gehrmann J, Raivich G, et al. MHC-positive, ramified macrophages in the normal and injured rat peripheral nervous system. $\mathcal{f}$ Neurocytol 1992;21: 623-34.

19 Kiefer R, Kieseier BC, Brück W, et al. Macrophage differentiation antigens in acute and chronic autoimmune polyneuropathies. Brain 1998;121:469-79.

20 Schmidt B, Toyka KV, Kiefer R, et al. Inflammatory infiltrates in sural nerve biopsies in Guillain-Barre syndrome and chronic inflammatory demyelinating neuropathy. Muscle Nerve 1996;19:474-87.

21 Asbury AK, Cornblath DR. Assessment of current diagnostic criteria for Guillain-Barré syndrome. Ann Neurol 1990; 27:S21-4.

22 Ad hoc subcommittee of the American Academy of Neurology AIDS task force. Research criteria for diagnosis of chronic inflammatory demyelinating polyneuropathy
(CIDP). Neurology 1991;41:617-18. 
23 Vyth Dreese FA, Dellemijn TA, Majoor D, et al. Localization in situ of the co-stimulatory molecules B7.1 B7.2, CD40 and tissue. Eur $\mathcal{F}$ Lmmun $1995 ; 25: 3023-9$.

24 Bourde O, Kiefer R, Toyka KV, et al. Quantification of interleukin- 6 mRNA in wallerian degeneration by competitive reverse transcription polymerase chain reaction. $f \mathrm{Neu}$ roimmunol 1996;69:135-40.

25 Meurers B, Kohlhepp W, Gold R, et al. Histopathological findings in the central and peripheral nervous system in neuroborreliosis. A report of three cases. $\mathcal{F}$ Neurol 1990;237:113-16.

26 Kreutzberg GW. Microglia: a sensor for pathological events in the CNS. Trends Neurosci 1996;19:312-318.

27 Issazadeh S, Navikas V, Schaub M, et al. Kinetics of expression of costimulatory molecules and their ligands in murine relapsing experimental autoimmune encephalomyelitis in vivo. F Immunol 1998;161:1104-12.

28 Thompson CB. Distinct roles for the costimulatory ligands B7-1 and B7-2 in T helper cell differentiation? Cell 1995; 81:979-82.

29 Yssel H, Shanafelt MD, Sodenberg C, et al. Borrelia burgdorferi activates a $\mathrm{T}$ helper type 1 -like $\mathrm{T}$ cell subset in burgdorferi activates a T helper type 1-like T
Lyme arthritis. $\mathcal{F}$ Exp Med 1991;174:593-601.

30 Matyniak JE, Reiner SL. T helper phenotype and genetic susceptibility in experimental Lyme disease. $\mathcal{F}$ Exp Med 1995;181:1251-4.
31 Pohl-Koppe A, Balashov KE, Steere AC, et al. Identification of a T cell subset capable of both IFN- $\gamma$ and IL-10 secretion in patients with chronic Immul 1998,15:1804-10.

32 Schmidt B, Stoll G, van der Meide PH, et al. Transient cellular expression of interferon- $\gamma$ in experimental autoimmune neuritis. Brain 1992;115:1633-46.

33 Stoll G, Jung S, Jander S, et al. Tumor necrosis factor- $\alpha$ in mmune mediated demyelination and Wallerian degeneration of the rat peripheral nervous system. F Neuroimmunol 1993;45:175-82.

34 Jander S, Pohl J, Gillen C, et al. Differential expression of interleukin-10 mRNA in Wallerian degeneration and immune-mediated inflammation of the rat peripheral nervous system. F Neurosci Res 1996;43:254-9.

35 Kiefer R, Funa K, Schweitzer T, et al. Transforming growth factor-b1 in experimental autoimmune neuritis: cellular localization and time course. Am f Pathol 1996;148:21123.

36 Zhu J, Bai XF, Mix E, et al. Cytokine dichotomy in peripheral nervous system influences the outcome of experimental allergic neuritis: dynamics of mRNA expression for IL-1 $\beta$, IL- 6, IL-10, IL-12, TNF- $\alpha$, TNF- $\beta$, and cytolysin. Clin $\beta$, IL- 6, IL-10, IL-12, TNF- $\alpha$, TNF- $\beta$,

37 Karandikar NJ, Vanderlugt CL, Bluestone JA, et al. Targeting the B7/CD28:CTLA-4 costimulatory system in CNS autoimmune disease. F Neuroimmunol 1998;89:10-18. 